\title{
Estimation of picked-up lunar ions for future compositional remote SIMS analyses of the lunar surface
}

\author{
Shoichiro Yokota $^{1 *}$ and Yoshifumi Saito ${ }^{1}$ \\ ${ }^{1}$ Institute of Space and Astronautical Science, Kanagawa 229-8510, Japan
}

(Received August 30, 2004; Revised February 1, 2005; Accepted March 10, 2005)

\begin{abstract}
In situ measurement of Moon-originating ions picked-up in orbit round the Moon is expected to provide valuable information regarding the thin lunar atmosphere and surface. Secondary ions sputtered by the solar wind ions reflect the surface abundance. Global composition mapping of the lunar surface may be thus achieved by measuring the sputtered ions as one would perform laboratory SIMS. We studied the dynamics of picked-up lunar ions when the Moon was exposed to the solar wind. Our model's source mechanism involved photoionization of the lunar exospheric atoms, photon-stimulated ion desorption, and ion sputtering. We propose that an intense flux of picked-up lunar ions $\left(10^{4} / \mathrm{cm}^{2} \mathrm{sec}\right)$ exists at an altitude of $100 \mathrm{~km}$, for nearly a quarter of the orbit. The ion flux originating from the lunar surface is mono-directional and mono-energetic, and is distinguishable from that of lunar atmospheric origin whose energy spectra correspond to their spatial distribution. Our calculation suggested that ion measurements in orbit round the Moon enable remote SIMS analyses.
\end{abstract}

Key words: Picked-up lunar ions, lunar surface, compositional remote SIMS analysis.

\section{Introduction}

The Moon, Mercury, and some of the planetary satellites and asteroids maintain thin and sometimes transient atmospheres. Of these bodies, the Moon and its atmosphere have been the most extensively investigated, and the findings are thought to be the cornerstone on which to build studies of the other celestial bodies. The lunar atmosphere is called 'surface-bounded exosphere' because it is thin enough to be regarded as an exosphere and it bounds on the solid surface differently from the Earth's atmosphere-bounded exosphere. Rare collisions among lunar atmospheric particles cause composite components that independently possess their own distribution, defined by their unique surface energy and abundance, mass, radiation pressure, and source and loss processes. Interactions between lunar atmospheric particles and the surface via collision processes, such as exchange of composition, thermal accommodation, chemical reactions and re-absorption, have large effects on the lunar atmosphere (Smyth and Marconi, 1995). Hence, the independent atmosphere presumably preserves information on the characteristics of the surface.

Sputtering caused by solar wind ions has especially attracted considerable attention, because it produces secondary particles reflecting the abundance of the lunar surface. Solar wind particles easily hit the lunar surface because the Moon has neither a global intrinsic magnetic field nor substantial atmosphere, unlike the Earth whose

*Present address: National Institute of information and Communications Technology, Tokyo 184-8795, Japan.

Copy right(c) The Society of Geomagnetism and Earth, Planetary and Space Sciences (SGEPSS); The Seismological Society of Japan; The Volcanological Society of Japan; The Geodetic Society of Japan; The Japanese Society for Planetary Sciences; TERRAPUB. magnetosphere provides an effective obstacle to solar wind plasma. Particles sputtered from the lunar surface by the solar wind are mainly composed of neutrals, which are ionized by solar photons and electrons. Both ionized particles and sputtered ions are transported by the solar wind in a cycloidal motion if the solar wind's electric and magnetic fields are steady and their initial energy is negligible. Although some of the ions picked-up by the solar wind hit the lunar surface and are reabsorbed, the others are driven into space. Managadze and Sagdeev (1988), Elphic et al. (1991) and Funsten et al. (1992) proposed that lunar orbiters might be useful for remotely performing global composition mappings by measuring ions sputtered by the solar wind and tracing them back to their sputtering point as in laboratory Secondary Ion Mass Spectrometry (SIMS).

Although many in situ measurements were done using spacecraft such as Apollo in the early days of space exploration, few clear detections of Moon-originating atoms and ions were obtained because the instruments were constrained by contamination and outgassing around them (e.g., Hodges et al., 1972; Hodges, 1975). The UV spectrometer on board Apollo 17 for detecting the resonance fluorescence lines of $\mathrm{H}, \mathrm{O}, \mathrm{C}, \mathrm{N}, \mathrm{Kr}, \mathrm{Xe}, \mathrm{H}_{2}$, and $\mathrm{CO}$ also showed no valid detection. Feldman and Morrison (1991) estimated the upper limits of the number density of these species based on the instrumental sensitivity. On the contrary, ground-based optical observations have succeeded in detecting the lunar alkali atmosphere. At the end of 1980s, $\mathrm{D}_{2}$-line emissions indicating the existence of $\mathrm{Na}$ and $\mathrm{K}$ atmospheres were discovered for the first time above the sunlit limb of the Moon (Potter and Morgan, 1988; Tyler et al., 1988). Lately, there has been observational evidence that solar photons play a dominant role in desorption of the lunar alkali atmosphere (Potter et al., 2000). Sputter- 
ing of the lunar surface has also been suggested to contribute subsidiarily to creation of the lunar $\mathrm{Na}$ atmosphere by enhancing diffusion of $\mathrm{Na}$ to the ground surface. Laboratory work also concluded that a photon-stimulated process could achieve a yield and kinetic temperature of the $\mathrm{Na}$ atmosphere consistent with previous observations (Madey et al., 1998; Yakshinskiy and Madey, 1999). On the other hand, optical observations by Flynn and Stern (1996) and by Stern et al. (1997) did not detect $\mathrm{Si}, \mathrm{Al}, \mathrm{Ca}, \mathrm{Mg}, \mathrm{Fe}$ and $\mathrm{Ti}$, or $\mathrm{Al}, \mathrm{Si}, \mathrm{Mg}$ and $\mathrm{Mg}^{+}$, respectively, and hence the researchers could only estimate the upper limits of their number densities. If the lunar atmosphere is stoichiometric relative to the lunar surface, the upper limits are much lower compared with the observed densities of $\mathrm{Na}$ and $\mathrm{K}$. Thus, $\mathrm{Na}$ and $\mathrm{K}$ might be regarded as peculiar species in the lunar atmosphere, which are preferably desorbed by solar photons, due to their volatility. Accordingly, a compositional analysis of the lunar surface made by measuring lunar atmospheric particles would require an evaluation of all the source processes, each of which does not necessarily reflect the surface abundance.

Solar wind particles undoubtedly sputter neutrals and ions of all species composing the lunar regolith with substantial fluxes (Elphic et al., 1991). The ion analyzer on board the AMPTE satellite detected singly ionized pickedup particles, which were supposed to be of lunar origin, upstream of the Earth's bow shock (Hilchenbach et al., 1993). Picked-up lunar ions have also been detected by the WIND spacecraft (Mall et al., 1998). The detection, which was attributed to $\mathrm{O}^{+}, \mathrm{Al}^{+}, \mathrm{Ca}^{+}, \mathrm{Fe}^{+}, \mathrm{Si}^{+}$and $\mathrm{P}^{+}$, suggested that lunar ions were sputtered by the solar wind, with large production rates that were roughly consistent with the results of Elphic et al. (1991). The ion transport simulation of Cladis et al. (1994) verified that some lunar ion species could be detected by spacecraft that is the upstream of the Earth's bow shock in spite of diffusion caused by magnetic turbulence.

These previous observations and experiments suggested that a variety of ions originating from the lunar atmospheres and surface presumably exist around the Moon. In situ measurements of these ions by a lunar orbiter can provide valuable information regarding the ionized lunar atmosphere and ions sputtered from the lunar surface. In this article, we study the dynamics of picked-up lunar ions and estimate their flux. To discuss the significance of in situ ion measurement, we assume there is a lunar orbiter at an altitude of 100 $\mathrm{km}$. Because the thin lunar atmosphere has little effect on the plasma interaction as long as they remain thin, we use a test particle model in which we derive the production rate of lunar ions from the results of the previous observations and laboratory experiments.

\section{Sources and Transport}

To estimate the flux of picked-up lunar ions, we investigated the production rates of various ions constituting the lunar atmosphere and soil. Lunar ions are mainly produced by desorption of the lunar soil constituents as ions and by ionization of atmospheric particles. After describing the yields for various species, we discuss the transport processes of picked-up lunar ions used in our test particle model.

\subsection{Desorption processes}

As for the source mechanism of the thin lunar alkali atmosphere, five processes have been proposed. These are (1) thermal desorption, (2) electron-stimulated desorption (ESD), (3) photon-stimulated desorption (PSD), (4) ioninduced desorption (sputtering), and (5) vaporization by micrometeoroid impacts (Madey et al., 1998, and references therein). The main product of all the source processes is neutral for almost all the species. Below, we will estimate the ion yields of these desorption processes. The results of this estimate indicated that we should use only the yields of PSD and sputtering for our model.

The ordinary alkali lunar atmosphere was suggested to be supplied by thermal desorption, ESD, PSD and/or sputtering because of the zenith angle dependence (Potter and Morgan, 1991), while micrometeoroid impacts episodically enhanced $\mathrm{Na}$ emission during meteor showers, such as the Leonids in 1997 (Cremonese and Verani, 1997; Smith et al., 1999). Thus, we neglected vaporization by micrometeoroid impacts when calculating the production rate. Since both observed $\mathrm{Na}$ and $\mathrm{K}$ atmospheres have non-thermal distribution, thermal desorption was thought to have little effect on the lunar atmosphere. Moreover, Madey et al. (1998) argued that the $\mathrm{Na}$ binding energy of $\sim 1.8 \mathrm{eV}$ corresponded to a temperature of $\sim 700 \mathrm{~K}$ and this temperature was higher than the temperature $(400 \mathrm{~K})$ at the lunar subsolar point. Accordingly, we also neglected ions produced by thermal desorption. ESD and PSD are electronic processes via subsequent intra-atomic Auger decay caused by incident electrons and photons, respectively (Ageev et al., 1995). Both of them preferably desorb alkali species whose binding energy to oxides is relatively small. The laboratory study of Yakshinskiy and Madey (1999) and the ground-based observation of Potter et al. (2000) confirmed that the lunar Na atmosphere was mainly maintained by PSD. In particular, the result of the laboratory experiment showed that ESD's contribution to the production rate was far less (Yakshinskiy and Madey, 1999). Moreover, a theoretical modeling of the $\mathrm{Na}$ exosphere indicated that PSD was a likely process on the basis of the exosphere's temperature (Smyth and Marconi, 1995). After considering these findings, we omitted ions desorbed by ESD from our calculation.

Our calculation used the production rates of lunar $\mathrm{Na}$ and K neutrals estimated by several optical observations (e.g., Potter and Morgan, 1998) to PSD and estimated the production rate of $\mathrm{Na}^{+}$and $\mathrm{K}^{+}$ions by PSD. The other species known to compose the lunar surface have not been (optically) found around the Moon. The upper limits of their number density estimated by Flynn and Stern (1996) and Stern et al. (1997) were lower than the stoichiometric value based on the observational density of the lunar atmospheric $\mathrm{Na}$ and $\mathrm{K}$ and the abundance of the lunar soil. Therefore, we assumed that only alkali species were desorbed by PSD. On the basis of the observations of Mendillo et al. (1991), Smyth and Marconi (1995) estimated the Na yield at the subsolar point at from $2.1 \times 10^{6}$ to $3.5 \times 10^{5} / \mathrm{cm}^{2} \mathrm{sec}$. Here, we used a PSD yield of $\mathrm{Na}$ equal to the lower limit of $3.5 \times 10^{5} / \mathrm{cm}^{2} \mathrm{sec}$ in order to determine whether the intensity of picked-up lunar ions is over the detectable level of 
space-borne ion analyzers. Although the PSD yield of $\mathrm{Na}^{+}$ ions is unknown, the ESD cross sections of Na neutrals and $\mathrm{Na}^{+}$ions have been measured by Ageev et al. (1995). The ESD cross-section ratio of $\mathrm{Na}^{+} / \mathrm{Na}$ is from $\sim 0.1$ to $\sim 0.001$ dependent on Na coverage on the oxide. A smaller coverage give a larger ESD cross section of $\mathrm{Na}^{+}$, while the cross section of $\mathrm{Na}$ is constant. Because PSD and ESD have the same electronic process (Madey et al., 1998), we supposed that their cross-section ratios of $\mathrm{Na}^{+} / \mathrm{Na}$ are the same. Considering the small abundance of alkali species in the lunar soil, we assumed that the ratio would be over 0.01 . In our calculation, we used a ratio equal to 0.01 . Accordingly, the PSD yield of $\mathrm{Na}^{+}\left(3.5 \times 10^{3} / \mathrm{cm}^{2} \mathrm{sec}\right)$ was obtained by multiplying the PSD yield of $\mathrm{Na}$ by the cross-section ratio of $\mathrm{Na}^{+} / \mathrm{Na}$. Note that the PSD yield of $\mathrm{K}$ was not calculated and the ESD cross sections of $\mathrm{K}$ and $\mathrm{K}^{+}$were not measured. We assumed that the PSD yield is proportional to the surface density and that the PSD cross-section ratio of $\mathrm{K}^{+} / \mathrm{K}$ is the same as that of $\mathrm{Na}^{+} / \mathrm{Na}$. Note that the decrease in the ESD cross section from $\mathrm{Li}^{+}$to $\mathrm{Cs}^{+}$(Ageev et al., 1995) suggests that the $\mathrm{K}^{+}$cross section is possibly smaller than that of $\mathrm{Na}^{+}$. The surface number density ratio of $\mathrm{Na} / \mathrm{K}$ ( 2.9) was derived from the observations of Sprague et al. (1992). The PSD yield of $\mathrm{K}^{+}$was thus taken to be $1.2 \times 10^{3}$ $/ \mathrm{cm}^{2} \mathrm{sec}$.

Table 1 shows the estimated production rates of $\mathrm{Na}^{+}$and $\mathrm{K}^{+}$ions due to PSD at the subsolar point. Since the production rates are proportional to the solar photon flux, the dependence on the solar zenith angle $\phi$ was $\cos \phi$ in our model. Moreover, Elphic et al. (1991) experiment suggested that there are substantial yields of various ions sputtered by the solar wind from the lunar surface. They reported secondary ion yields caused by $\mathrm{H}^{+}, \mathrm{He}^{++}$and $\mathrm{Ar}^{+}$ bombardments of lunar soil simulants. We used the solar wind sputtering yields as those at the subsolar point (Table 1). The dependence on the solar zenith angle was $\cos \phi$ because the sputtering yields are proportional to the solar wind ion flux.

\subsection{Ionization of the lunar alkali exosphere}

We also considered lunar ions produced by ionization processes from lunar atmospheric particles. Since only $\mathrm{Na}$ and $\mathrm{K}$ species have been found by optical measurement, we assumed that only alkali atmospheres exist around the Moon. The spatial distribution of their number densities was studied as follows. Potter and Morgan (1998) showed the uniformity of the dayside surface number density over the dayside zenith angle. They also indicated a strong dependence of vertical column abundance on zenith angle, which was fitted to a cosine-squared curve. Based on the observational results, we calculated the number density $n_{i}(h, \phi)$ of the alkali atmosphere $i$ at an altitude $z$ and a zenith angle $\phi$ by using the geopotential altitude $z_{g p}$ (Sprague et al., 1992). $n_{i}(h, \phi)$ is given by

$$
\begin{aligned}
n_{i}(z, \phi) & =n_{i, 0} \cdot \exp \left(-\frac{z_{g p}}{H_{i}^{*}}\right), \\
z_{g p} & \equiv \frac{z R_{m}}{z+R_{m}},
\end{aligned}
$$

where $n_{i, 0}, H_{i}^{*}$ and $R_{m}$ denote the number density on the lunar surface at the subsolar point, apparent scale height and
Table 1. Yields of ions from the lunar surface desorbed by PSD and sputtering. The PSD yields of $\mathrm{Na}^{+}$and $\mathrm{K}^{+}$were computed by using the PSD yields of $\mathrm{Na}$ and $\mathrm{K}$ and the PSD cross-section ratios of $\mathrm{Na}^{+} / \mathrm{Na}$ and $\mathrm{K}^{+} / \mathrm{K}$. The PSD yield of $\mathrm{Na}$ is derived from Smyth and Marconi (1995) calculation which agrees with the observation of Mendillo et al. (1991). The PSD yield of K is derived from the PSD yield of Na and the density ratio of $\mathrm{Na} / \mathrm{K}$ observed by Sprague et al. (1992). The PSD cross-section ratios of $\mathrm{Na}^{+} / \mathrm{Na}$ and $\mathrm{K}^{+} / \mathrm{K}$ are assumed to be the same as the ESD cross-section ratio of $\mathrm{Na}^{+} / \mathrm{Na}$ measured by Madey et al. (1998). The PSD yields other than $\mathrm{Na}^{+}$and $\mathrm{K}^{+}$are ruled out in our model. The sputtering yields are derived from the experimental result of Elphic et al. (1991)). Both PSD and sputtering yields are at the subsolar point. These yields have the solar zenith angle dependence expressed as $\cos \phi$, where $\phi$ is the zenith angle.

\begin{tabular}{rrr}
\hline Species & $\begin{array}{c}\text { PSD } \\
\left(/ \mathrm{cm}^{2} \mathrm{sec}\right)\end{array}$ & $\begin{array}{c}\text { Sputtering } \\
\left(/ \mathrm{cm}^{2} \mathrm{sec}\right)\end{array}$ \\
\hline $\mathrm{Na}^{+}$ & $3.5 \times 10^{3}$ & $2.4 \times 10^{3}$ \\
$\mathrm{Mg}^{+}$ & - & $2.8 \times 10^{3}$ \\
$\mathrm{Al}^{+}$ & - & $6.0 \times 10^{3}$ \\
$\mathrm{Si}^{+}$ & - & $2.0 \times 10^{3}$ \\
$\mathrm{~K}^{+}$ & $1.2 \times 10^{3}$ & $9.5 \times 10^{2}$ \\
$\mathrm{Ca}^{+}$ & - & $6.5 \times 10^{3}$ \\
$\mathrm{Ti}^{+}$ & - & $3.0 \times 10^{2}$ \\
$\mathrm{Mn}^{+}$ & - & $2.5 \times 10^{1}$ \\
$\mathrm{Fe}^{+}$ & - & $8.5 \times 10^{2}$ \\
\hline
\end{tabular}

lunar radius $(1738 \mathrm{~km})$, respectively. Since the dependence of the vertical column abundance on the zenith angle $\phi$ directly corresponds to the scale height $H_{i}^{*}, H_{i}^{*}$ is described as

$$
H_{i}^{*}(\phi)=H_{i, 0}^{*} \cdot \cos ^{2} \phi
$$

where $H_{i, 0}^{*}$ is apparent scale height at the subsolar point. $n_{i, 0}$ and $H_{i, 0}^{*}$ were derived from the observations of Potter and Morgan (1998) for $i=\mathrm{Na}$ and Sprague et al. (1992) for $i=\mathrm{K}$. All the values are listed in Table 2 .

For the $\mathrm{Na}$ and $\mathrm{K}$ species, photoionization of lunar atmospheric particles far exceed charge exchange with the solar wind ions or electrons around the Moon. Note that photoionization is the dominant loss process of the lunar atmosphere for all species except light species such as $\mathrm{H}$ and He (Killen and Ip, 1999). We calculated the yield $Y_{i}$ for $\mathrm{Na}^{+}$and $\mathrm{K}^{+}(i=\mathrm{Na}$ and $\mathrm{K})$ by using the spatial distribution expressed by Eq. (1) and photoionization lifetime $T_{i}$ for $i=\mathrm{Na}$ and K listed by Killen and Ip (1999) as follows,

$$
Y_{i}(z, \phi)=n_{i}(z, \phi) \cdot \frac{1}{T_{i}} .
$$

The values of $T_{i}$ for $i=\mathrm{Na}$ and $\mathrm{K}$ used in this calculation are also listed in Table 2. Our calculation excluded other ionization processes whose lifetimes were much longer.

\subsection{Transport}

Our calculation assumed that the Moon was immersed in a typical solar wind with a velocity $\boldsymbol{V}_{S W}$ of $400 \mathrm{~km} / \mathrm{sec}$ and magnetic field $\boldsymbol{B}_{S W}$ of $5 \mathrm{nT}$. Just after the lunar ions have been produced, they are affected by the local magnetic field $\boldsymbol{B}$ which is mainly attributed to the interplanetary magnetic field (IMF) $\left(\boldsymbol{B} \sim \boldsymbol{B}_{S W}\right)$, and the local electric field expressed as $\boldsymbol{E}=-\boldsymbol{V}_{S W} \times \boldsymbol{B}(\sim 2 \mathrm{mV} / \mathrm{m})$ in the frame of the Moon. The gravity of the Moon and the solar radiation pressure negligibly affect ion transport in comparison with 
Table 2. Characteristics of the lunar alkali atmospheres. The number density and apparent scale height are cited from Potter and Morgan (1998) for Na and Sprague et al. (1992) for K. The photoionization lifetime is cited from Killen and Ip (1999).

\begin{tabular}{cccc}
\hline Species & $\begin{array}{c}\text { Number density } \\
\left(/ \mathrm{cm}^{3}\right)\end{array}$ & $\begin{array}{c}\text { Apparent scale } \\
\text { height }(\mathrm{km})\end{array}$ & $\begin{array}{c}\text { Photoionizatin } \\
\text { lifetime }(\mathrm{sec}) \\
H_{i, 0}^{*}\end{array}$ \\
\hline $\mathrm{Na}$ & 35 & 510 & $T_{i}$ \\
$\mathrm{~K}$ & 10 & 85 & $6.2 \times 10^{4}$ \\
\hline
\end{tabular}

the electromagnetic forces, although they play an important role in the transport of lunar exospheric atoms. For $\mathrm{Na}^{+}$, the lunar surface gravity, the radiation pressure at a nominal heliocentric distance of $1 \mathrm{AU}$ and the local electric field of $2 \mathrm{mV} / \mathrm{m}$ are $1.622,2.71 \times 10^{-2}$ and $4.41 \times 10^{6} \mathrm{~m} / \mathrm{s}^{2}$, respectively. A magnetic perturbation around the Moon exists, and it originates from the solar wind itself or from the interaction between the Moon and the solar wind. The wide-area ion transport simulation of Cladis et al. (1994) involved an IMF perturbation, which was generated by the method of Price and Wu (1987) and Terasawa (1989). However, the typical hydromagnetic perturbation estimated by Coleman (1968) and Denskat et al. (1983) is weak and leisurely enough to be neglected because the picked-up lunar ions' flight time and course between their desorption areas and detection point are short $(\sim 1 \mathrm{sec}$ and $\sim 100 \mathrm{~km})$. Thus, our calculation first omitted the perturbation of the IMF. After showing the calculation results without the perturbation of the IMF, we will discuss the effects of the perturbation on the ion transport in detail.

A body like the Moon without a global magnetic field or substantial atmosphere merely obstructs and absorbs the solar wind plasma that is incident on it. Because the IMF rapidly diffuses into the very weak conducting layers, neither a bow shock nor a magnetosphere like those of the Earth is formed in front of the Moon. Although the lunar conductive core should magnetically perturb the field lines threading the body, no large perturbation has been found yet. Thus, we assumed that the magnetic perturbation induced by the conductive core of the Moon is negligible.

Some of the remnant magnetisms near the lunar surface, however, form localized dipolar arches of magnetic fields that extend into the solar wind. The global distribution and structure of the magnetic anomalies on the lunar surface were studied by the Lunar Prospector (e.g., Lin et al., 1998; Halekas et al., 2001). Large magnetic anomalies are a few 100s of $\mathrm{km}$ in scale and a few 100s of nT in intensity. These magnetic anomalies presumably block the solar wind and stop lunar ions from being picked-up by it. However, because the most of the lunar surface is unaffected by these local anomalies, our calculation ignored them. In a future orbital investigation of the lunar surface and atmosphere, we will consider the effect of magnetic anomalies. In summary, to simulate the typical solar wind, we ignored all magnetic perturbations and assumed that the IMF and the velocity of the solar wind are steady with respect to time and space $\left(\boldsymbol{B}_{S W}=\boldsymbol{B}_{0}, \boldsymbol{V}_{S W}=\boldsymbol{V}_{0}\right)$.

The measured lunar surface electric potential is about 10 $\mathrm{V}$ on the dayside and of $-10 \mathrm{~V}$ to $-100 \mathrm{~s} \mathrm{~V}$ on the nightside, and it is due to the emission of photoelectrons and in- trusion of surrounding thermal electrons (see, e.g., Freeman and Ibrahim, 1975). In our calculation, the potentials of the subsolar surface and its antipode were 10 and $-100 \mathrm{~V}$, respectively. The zenith angle dependence of the dayside and nightside was $|\cos \phi|$. The dayside and nightside potential layers were $100 \mathrm{~m}$ thick, the same thickness as the dayside photoelectron sheath as reported by Walbridge (1973). The electric potential of the lunar surface was superposed on the surrounding electric fields.

In the case of $\mathrm{Na}^{+}$after desorption or photoionization, initial energy of lunar ions before being accelerated by the surrounding electric fields is up to a few eV corresponding to a few $\mathrm{km} / \mathrm{sec}$, which is much smaller than the energy supplied by the surrounding electric field. Hence, we ignored the initial energy of lunar ions.

The equation of motion for lunar ions is

$$
m \frac{d \boldsymbol{v}}{d t}=q(\boldsymbol{E}+\boldsymbol{v} \times \boldsymbol{B}),
$$

where $m, v$ and $q$ are the mass, velocity vector and charge of ions, respectively. This equation was solved numerically with the Runge-Kutta algorithm in a situation where the upstream IMF is perpendicular to the flow of the solar wind. We chose the coordinates in such a way that the upstream solar wind flowed in the $-x$ direction, the upstream magnetic field was in the $+y$ direction, and the $z$-axis constituted a right-handed coordinate system.

\section{Results}

Here, we show the results concerning $\mathrm{Na}^{+}$ions pickedup by the solar wind because the $\mathrm{Na}$ atmosphere is the most conspicuous of all the species. As described above, $\mathrm{Na}^{+}$ ions are generated mainly by three processes; photoionization of the Na atmospheric particles, PSD, and solar wind sputtering. Figure 1 shows the calculated yields per unit volume of $\mathrm{Na}^{+}$ion originating from the lunar atmosphere and the surface along the Sun-Moon line. Although the yields of $\mathrm{Na}^{+}$generated by PSD (asterisk) and by the solar wind sputtering (triangle) are extremely larger than the yield of $\mathrm{Na}^{+}$originating from the atmosphere (solid line), the sum of the $\mathrm{Na}^{+}$yield from the atmosphere between the surface and $100-\mathrm{km}$ altitude is larger than the yields from the surface.

Figure 2 shows representative trajectories of picked-up ions emitted from the lunar surface on the $x-z$ plane perpendicular to the magnetic field. The electric field expressed as $\boldsymbol{E}=-\boldsymbol{V}_{S W} \times \boldsymbol{B}_{S W}$ is toward the $z$-axis. The trajectories of ions generated from the lunar atmosphere are not shown for simplicity. The solid circle indicates the lunar surface, and the $100-\mathrm{km}$ altitude almost equals the line 


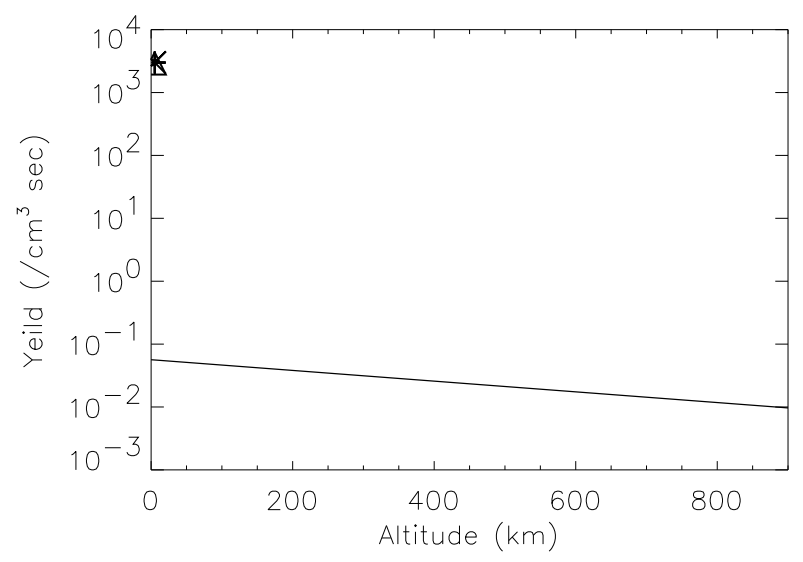

Fig. 1. $\mathrm{Na}^{+}$ion production rates per unit volume (ions $/ \mathrm{cm}^{3} \mathrm{sec}$ ) along the Sun-Moon line. The solid line denotes the Na atmosphere of photoionization origin. The asterisk and triangle denote the atmospheres of PSD origin and sputtering origin, respectively. Both PSD and sputtering have values only at unit volume adjacent to the surface.

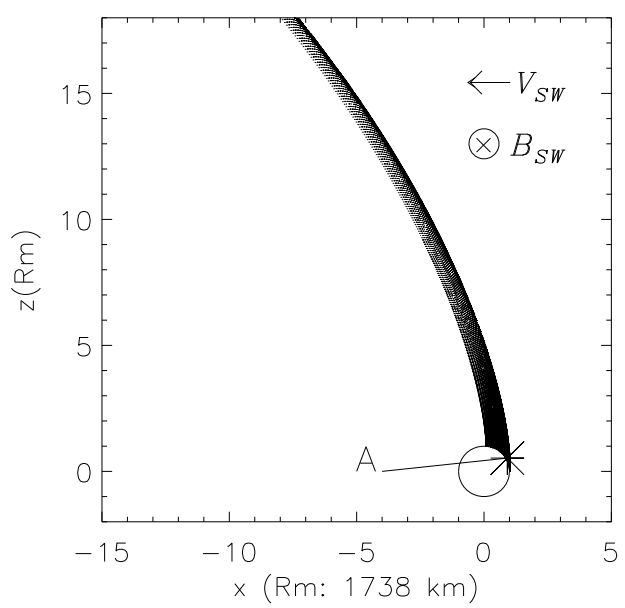

Fig. 2. Trajectories of picked-up $\mathrm{Na}^{+}$ions projected in the $\mathrm{x}-\mathrm{z}$ plane. The sampled ions are desorbed from the lunar surface. The circle indicates the lunar surface, and $100 \mathrm{~km}$ altitude almost equals the line thickness. Point A (asterisk) is located at an altitude of $100 \mathrm{~km}$ and a zenith angle of $30^{\circ}$. The upstream solar wind flows in the $-x$ direction, the upstream magnetic field is in the $+y$ direction, and the electric field is toward the $z$-axis constituting the right-handed coordinate system.

thickness. Ions emerge only from the dayside of the Moon, and approximately half of them escape outward. The rest of them fall on the lunar surface. As a result, a lunar orbiter can detect picked-up ions generated at the surface only during a quarter of its orbit. Because the picked-up $\mathrm{Na}^{+}$ions have a Larmor radius much larger than the radii of the Moon and the orbit at 100-km altitude, their motion from production point to $100-\mathrm{km}$ altitude follows nearly a straight line along the electric field direction. To investigate the pickedup $\mathrm{Na}^{+}$ion flux that a lunar orbiter would encounter, we set the representative point labeled as 'A' (see Fig. 2). Point A is located at the center of the large asterisk, at an altitude of $100 \mathrm{~km}$ and a zenith angle of $30^{\circ}$.

Picked-up ion energy, which is supplied mainly by the solar wind electric field, is approximately proportional to the ion path length, provided that the surrounding electric field is nearly constant. Figure 3 plots energy spectra of

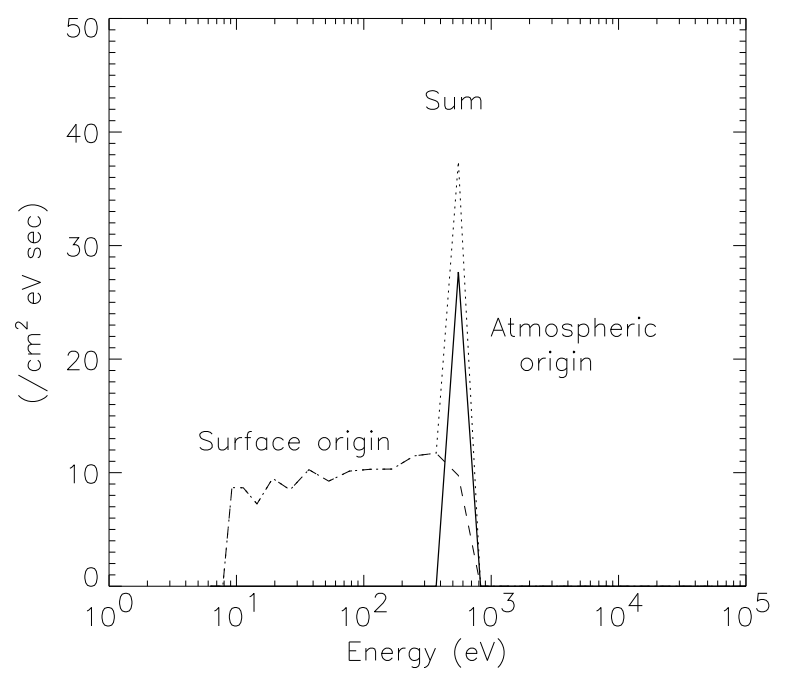

Fig. 3. Energy spectra of picked-up lunar $\mathrm{Na}^{+}$ions at point A (see Fig. 2). Solid and dashed lines denote ions of surface origin and atmospheric origin, respectively. The dotted line denotes the sum of surface origin and atmospheric origin.

the picked-up $\mathrm{Na}^{+}$ions of atmospheric origin (dashed line) and surface origin (solid line) passing through the point $\mathrm{A}$ (see Fig. 2). The dotted line denotes the sum of the spectra. Ions emitted from the surface are mono-energetic, and ions produced by photoionization from the $\mathrm{Na}$ atmosphere have much a wider energy distribution in the lower energy range. A composition mapping of the lunar surface by remote SIMS analysis requires the ability to discriminate ions of surface origin from ions of atmospheric origin. The energy supplied to picked-up ions depends on the potential difference between the production point of the ions and orbit, if the potential of the orbiter is assumed to be ground level. In other words, the energy measured in orbit indicates the production spot of these ions. Thus, energy spectra can be used to discriminate the surface originating ions from those of atmospheric origin. Moreover, the spectra correspond to the number density distribution of the lunar atmosphere. Note that energy spectra cannot be used for distinguishing between ions generated by PSD and by sputtering because these ions have the same spectra. Although there is a difference in their initial energy distribution (Smyth and Marconi, 1995), it is far less than the energy supplied by the surrounding electric field.

In the steady-state model, picked-up $\mathrm{Na}^{+}$ion motions are confined to the $x-z$ plane. Figure 4 shows that the distribution of the ion traveling direction at point A (see Fig. 2) is very narrow. Zero and $90^{\circ}$ correspond to the $x$ axis (anti-ward the solar wind) and the $z$-axis (toward the electric field expressed as $\boldsymbol{E}=-\boldsymbol{V}_{S W} \times \boldsymbol{B}_{S W}$ ), respectively. The solid line denotes the sum of surface and atmospheric originating ions. All the ions generated at different spots by different source processes travel along the electric field until they reach an altitude of $100 \mathrm{~km}$, due to the fact that the flight length is far shorter than the Larmor radius of picked up $\mathrm{Na}^{+}$ions $\left(\sim 2.0 \times 10^{4} \mathrm{~km}\right)$. Even the Larmor radius of picked-up $\mathrm{H}^{+}$ions is larger than $100 \mathrm{~km}$. Considering the energy spectra and the distribution of the direction angle of the picked-up $\mathrm{Na}^{+}$ions sampled at point A (see Fig. 2), it 


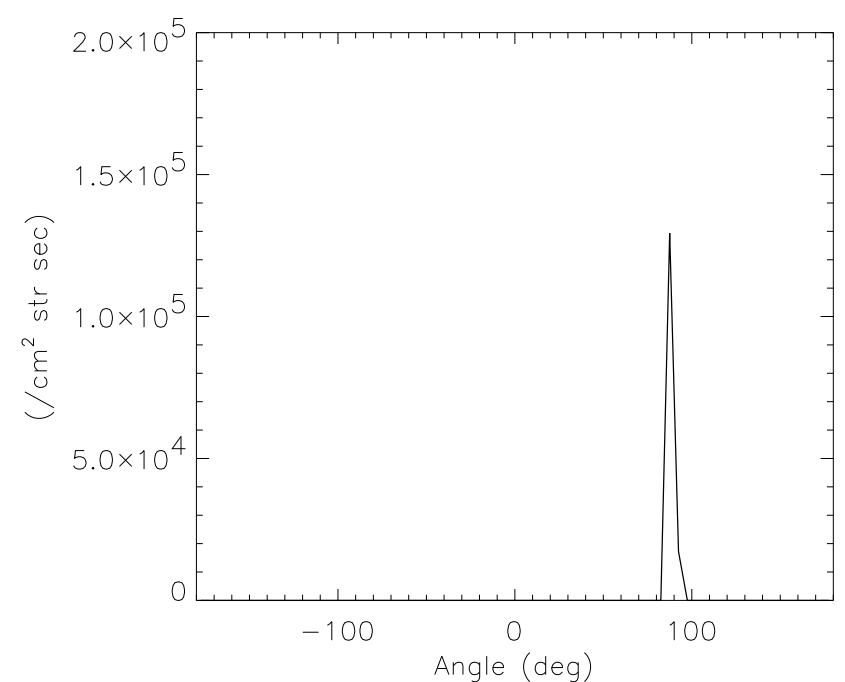

Fig. 4. Distribution of the traveling direction of picked-up lunar $\mathrm{Na}^{+}$ions at point A (see Fig. 2). A solid line denotes the sum of surface origin and atmospheric origin. The ion motion is confined to the $x-z$ plane. 0 and $90^{\circ}$ correspond to the $x$ direction (anti-ward the solar wind) and the $z$ direction (toward the electric field), respectively.

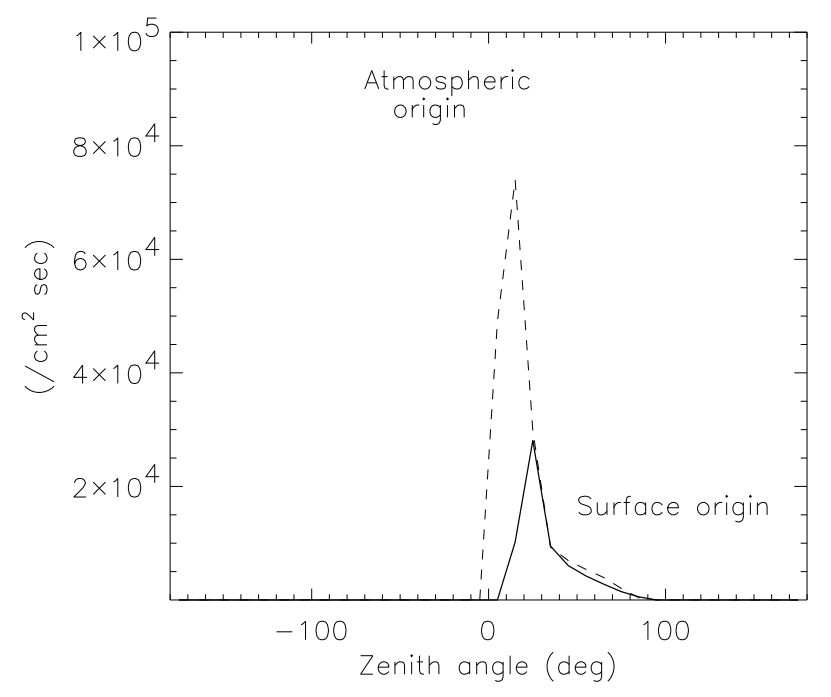

Fig. 5. Intensity of picked-up lunar $\mathrm{Na}^{+}$ions over the course of one orbit at $100-\mathrm{km}$ altitude. A positive value of zenith angle indicates that $\mathrm{z}$ component of the position in orbit is positive. Solid and dashed lines denote ions of surface origin and atmospheric origin, respectively. The motion of picked-up ions is almost mono-energetic and mono-directional for all species throughout the $100-\mathrm{km}$ altitude.

is clear that a mono-energetic and mono-directional flux is dominant at $100-\mathrm{km}$ altitude.

We estimated the flux intensity of picked-up $\mathrm{Na}^{+}$ions in the $x-z$ plane over the dayside orbit of the Moon at $100-\mathrm{km}$ altitude. Ions emitted from the lunar surface can be detected at $100-\mathrm{km}$ altitude only within zenith angles of $\sim 0^{\circ}$ to $90^{\circ}$, as shown in Fig. 5. Solid and dashed lines denote the surface and atmospheric originating ions, respectively. The ion flux intensity of atmospheric origin is larger than or comparable to that of lunar surface origin, and both of fluxes become weaker at zenith angles close to $90^{\circ}$. A detectable flux of $\mathrm{Na}^{+}$, at least $10^{4} / \mathrm{cm}^{2} \mathrm{sec}$, persists over nearly a quarter orbit. A recent space-borne ion analyzer of Yokota

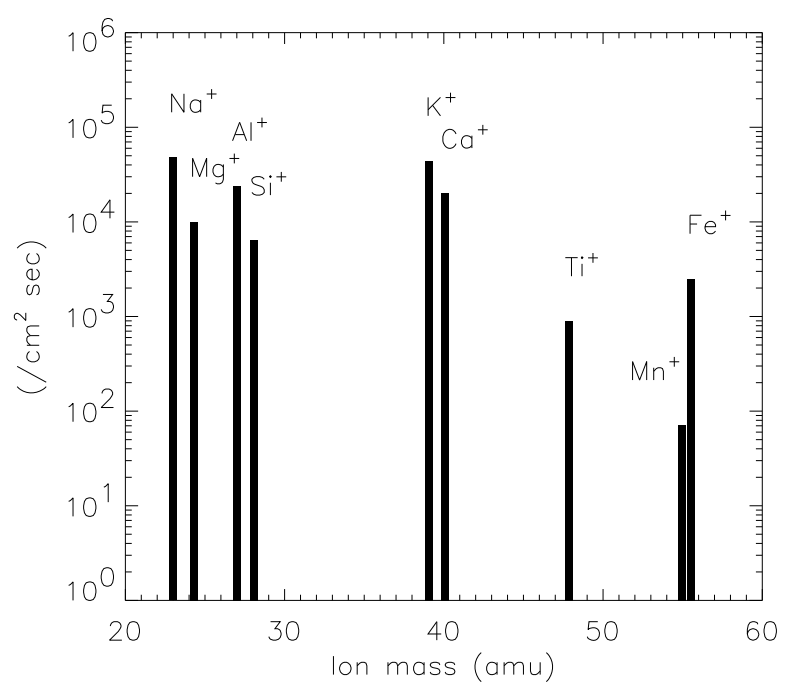

Fig. 6. Intensity of picked-up ions for $\mathrm{Na}^{+}, \mathrm{Mg}^{+}, \mathrm{Al}^{+}, \mathrm{Si}^{+}, \mathrm{K}^{+}, \mathrm{Ca}^{+}$, $\mathrm{Ti}^{+}, \mathrm{Mn}^{+}$, and $\mathrm{Fe}^{+}$at point A (see Fig. 2). All species include those of surface origin, whereas only $\mathrm{Na}^{+}$and $\mathrm{K}^{+}$contain those of atmospheric origin. The motion of picked-up ions for each species is almost mono-energetic and mono-directional for all species.

et al. (2005) can derive a hundredth counts in one minute from an ion flux of $10^{4} / \mathrm{cm}^{2} \mathrm{sec}$ within narrow energy and traveling direction ranges. For the remote SIMS analyses, a sampling time of one minute gives a spatial resolution of $100 \mathrm{~km}$ on the lunar surface, because lunar orbiters at 100$\mathrm{km}$ altitude move about $100 \mathrm{~km}$ per minute. Mass spectra which is obtained at point A (Fig. 2) are plotted in Fig. 6. The flux of each species is also mono-energetic and monodirectional. The $\mathrm{Mg}^{+}, \mathrm{Al}^{+}, \mathrm{Si}^{+}, \mathrm{K}^{+}, \mathrm{Ca}^{+}$and $\mathrm{Fe}^{+}$fluxes at $100-\mathrm{km}$ altitude are also statistically significantly intense. The $\mathrm{Ti}^{+}$flux at $100-\mathrm{km}$ altitude is at the level of one count per minute for the ion analyzers.

A spatially and temporally constant magnetic field was used in our calculation because hydromagnetic turbulence is small in the typical solar wind. Although some sorts of the Alfvénic turbulence show large fluctuations and they have nearly the same period as the flight time from the surface to $100-\mathrm{km}$ altitude ( $\sim 1 \mathrm{sec})$, they do not occur frequently. Here, we calculated the trajectories of picked-up lunar ions for such magnetic fluctuation. The average spectral density $P(f)\left(\mathrm{nT}^{2} / \mathrm{Hz}\right)$ of total power at the heliocentric distance of $0.87 \mathrm{AU}$ in magnetic field components measured in the trailing edges of high-speed streams is given by

$$
\log P(f)=-1.4 \log f-0.9,
$$

where $f$ denotes frequency (Hz) (Bavassano et al., 1982). By solving this equation discretely, we computed the fluctuation component $\tilde{B}(\mathrm{nT})$ expressed as

$$
\begin{aligned}
& \tilde{B}_{x}=\sum_{f=0.01}^{100} 0.3548 \times f^{-0.7} \sin \left(k y-\omega t+\theta_{f}\right)(\Delta f)^{0.5} \\
& \tilde{B}_{z}=\sum_{f=0.01}^{100} 0.3548 \times f^{-0.7} \cos \left(k y-\omega t+\theta_{f}\right)(\Delta f)^{0.5}
\end{aligned}
$$

where the initial phases $\theta_{f}$ are determined by a random number generator and the wavenumber $k$ and the angu- 

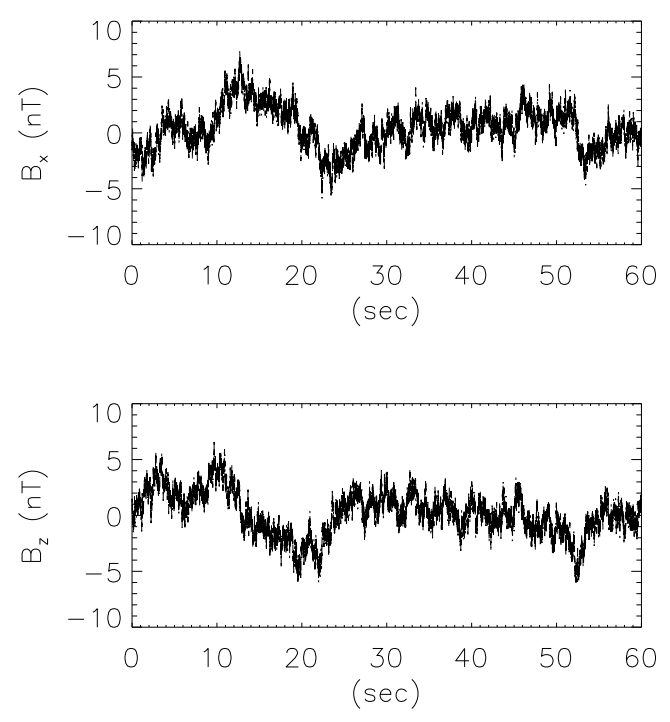

Fig. 7. Magnetic field in the $x-z$ plane $(y=0)$ between $t=0$ and $60 \mathrm{sec}$. Upper and lower panels show $B_{x}$ and $B_{z}$, respectively. $B_{y}$ is constant $\left(B_{0}=1 \mathrm{nT}\right)$. The Alfvén velocity $V_{A}$ is $65.5 \mathrm{~km} / \mathrm{sec}$.

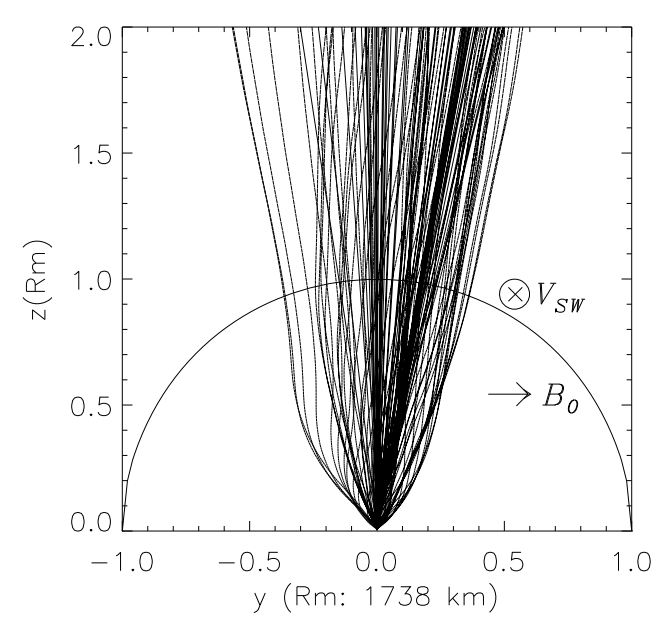

Fig. 8. Trajectories of picked-up $\mathrm{Na}^{+}$ions projected in the $y-z$ plane. The sampled ions are desorbed from the subsolar point of the Moon at $t=0,1,2, \ldots, 60 \mathrm{sec}$. The solid arc indicates the lunar surface. The upstream solar wind flows in the $-x$ direction, the constant component of the upstream magnetic field is toward the $+y$ direction, and the electric field is toward the $z$-axis constituting a right-handed coordinate system.

lar frequency $\omega=2 \pi f$ satisfy the hydromagnetic dispersion relation, $\omega= \pm k V_{A}$, where $V_{A}$ is the Alfvén velocity. $f$ contains a sufficiently high band compared with the ion flight time $(\sim 1 \mathrm{sec})$. The difference from the previous steady-state model was the solar wind magnetic field $\boldsymbol{B}_{S W}=\tilde{B}_{x} \boldsymbol{e}_{x}+B_{0} \boldsymbol{e}_{y}+\tilde{B}_{z} \boldsymbol{e}_{z}$ and the local electric field $\boldsymbol{E}=-\boldsymbol{V}_{S W} \times \boldsymbol{B}_{S W}$. The $x$ and $z$ components of the magnetic field in the $x-z$ plane $(y=0)$ between $t=0$ and $60 \mathrm{sec}$ are shown in Fig. 7. The magnetic field fluctuates a few $\mathrm{nT}$ in one second. We used the constant component of the magnetic field $B_{0}$ equal to $1 \mathrm{nT}$ instead of $5 \mathrm{nT}$, in order to emphasize the effect of the magnetic fluctuation on the ion trajectories. The Alfvén velocity $V_{A}$ was $65.5 \mathrm{~km} / \mathrm{sec}$. Figure 8 shows trajectories of picked-up ions projected in the $y-z$ plane. The ions were emitted only from the subsolar surface at $t=0,1,2, \ldots, 60 \mathrm{sec}$. Since the solar wind velocity $\boldsymbol{V}_{S W}$ is constant toward the $-x$ direction, the ion dispersion is mostly confined to the $y-z$ plane. Although there are a variety of ion trajectories, all the picked-up ions move nearly straight along the transient electric field until they reach $100-\mathrm{km}$ altitude. The flight time to $100-\mathrm{km}$ altitude is too short ( $\sim 1 \mathrm{sec}$ ) for the ions to substantially feel the magnetic fluctuation. The instantaneous flux of picked-up lunar ions at $100-\mathrm{km}$ altitude is almost mono-energetic and monodirectional, as in the steady-state model. Therefore, fast sampling theoretically enables tracing back to the desorption points on the lunar surface. However, one minute sampling gathers picked-up lunar ions with a variety of energies and motion directions. This suggests that if the sampling rate is low ( $\sim 1 \mathrm{~min})$, Alfvénic fluctuations may worsen the discrimination between ions of the atmospheric origin and surface origin and the spatial resolution of the composition mapping. In future remote SIMS analyses, if necessary, we should exclude the data with such magnetic turbulence.

\section{Discussion}

Our calculation showed that a variety of secondary ions composing the lunar soil exist at $100-\mathrm{km}$ altitude above the Moon at an intensity of $10^{4} / \mathrm{cm}^{2} \mathrm{sec}$ when the Moon is exposed to the solar wind. We showed that the flux of these ions at $100-\mathrm{km}$ altitude is almost mono-energetic and mono-directional. In addition, the ion flux of lunar surface origin is quite mono-energetic, and thus is distinguishable from that of lunar atmospheric origin whose energy spectra correspond to their spatial distribution. Figure 9 compares the sputtering yields at the subsolar point of Table 1 and the intensity of the picked-up ions of Fig. 6 for $\mathrm{Na}^{+}, \mathrm{Mg}^{+}, \mathrm{Al}^{+}$, $\mathrm{Si}^{+}, \mathrm{K}^{+}, \mathrm{Ca}^{+}, \mathrm{Ti}^{+}, \mathrm{Mn}^{+}$, and $\mathrm{Fe}^{+}$. Except for $\mathrm{Na}^{+}$and $\mathrm{K}^{+}$, the abundances of the sputtered ions have a constant ratio

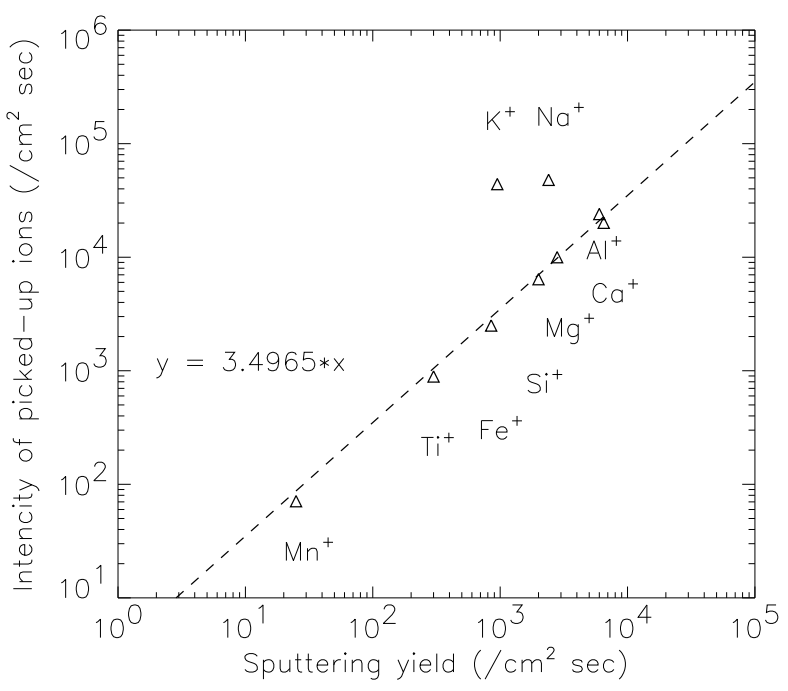

Fig. 9. Comparison of the sputtering yields of Table 1 and the intensity of the picked-up ions of Fig. 6 for $\mathrm{Na}^{+}, \mathrm{Mg}^{+}, \mathrm{Al}^{+}, \mathrm{Si}^{+}, \mathrm{K}^{+}, \mathrm{Ca}^{+}, \mathrm{Ti}^{+}$, $\mathrm{Mn}^{+}$, and $\mathrm{Fe}^{+}$. The vertical axis shows the intensity of the picked-up ions at point A (see Fig. 2), whose flux is almost mono-energetic and mono-directional. The horizontal axis shows the sputtering yields at the subsolar point. The broken line was computed using a least-squares program excluding $\mathrm{Na}^{+}$and $\mathrm{K}^{+}$. 
until 100-km altitude. Depending on the angle between the surface and the surrounding electric field, their flux reaches up to 3.5 times the sputtering yields. The flux of $\mathrm{Na}^{+}$and $\mathrm{K}^{+}$at $100-\mathrm{km}$ altitude includes the ions produced by PSD and photoionization of the lunar exosphere. It is not possible to directly identify the sputtered $\mathrm{Na}^{+}$and $\mathrm{K}^{+}$ among the ions of surface origin. However, we can estimate the lunar exosphere density profile by measuring energy spectra of the lunar ions and thus compute the PSD yield of $\mathrm{Na}$. By using the cross-section ratio of $\mathrm{Na}^{+} / \mathrm{Na}$, we can also estimate the PSD yield of $\mathrm{Na}^{+}$. Therefore, the sputtering yields of $\mathrm{Na}^{+}$and $\mathrm{K}^{+}$can be estimated as well as the other species. The picked-up lunar ion flux at $100-\mathrm{km}$ altitude can be used to estimate the sputtering yields for every species, which reflect the lunar surface abundance.

The yields of sputtered ions used in the calculation were those obtained in the laboratory lunar soil simulants experiment of Elphic et al. (1991). There has been no clear orbital detection of such sputtered ions. The WIND satellite measurements obtained the mass spectra of picked-up ions which were thought to originate from the Moon at $17 \mathrm{Rm}$ downstream with an intense flux of $\mathrm{O}^{+}$ions and a relatively faint flux of lunar alkali ions (Mall et al., 1998). However, optical observations confirmed large yields of alkali species (e.g., Potter and Morgan, 1998) and gave a relatively small number density of O atoms (Fleldman and Morrison, 1991). The mass spectra of the WIND satellite are inconsistent with these optical observations. As of yet, the experimental results of Elphic et al. (1991) are the only clues to the yields of sputtered lunar ions.

To investigate the lunar surface composition by measuring picked-up lunar ions at 100-km altitude, we need a precise estimate of secondary positive ion yields. The application of the Saha equation describing the charge state distribution probabilities of plasma is efficacious to quantitative SIMS analyses (Andersen and Hinthorne, 1972). In laboratory quantitative SIMS analysis, however, the relative sensitivity to the standard sample is generally utilized because the interaction between secondary ions and their matrix, which is called the 'matrix effect', strongly affects the absolute yield of secondary ions. Future remote SIMS analysis in orbit round the Moon will require the Saha equation, the absolute yield values obtained from laboratory experiments, or calibration using the compositional information of the Apollo landing sites as standard samples.

Optical spectrometers on board lunar orbiters such as Apollo, Clementine, and Lunar Prospector and groundbased telescopes have studied the formation, geologic history, composition, and resources of the Moon. These studies using wide mapping data revealed that the Moon has an intricate and globally diverse structure, and that not a few areas are compositionally different from the returned samples (Ben et al., 2000; Gaddis et al., 2000). The compositional data are important for characterizing the regolith, particularly in the study of crater ejecta distribution and large pyroclastic deposits. SIMS analysis, which can give detailed compositional information on the sample rock or soil similar to X-ray, gamma-ray, and near-infrared ray analyses, has never been applied to the remote measurement of the lunar surface. The advantages of remote SIMS analy- sis are its excellent sensitivity and accuracy to almost all species except rare gases due to the direct detection of ions sputtered from the sample materials. X-ray spectrum analysis of the lunar soil has difficulty in detecting alkali species because of their small compositional ratio and weak fluorescence yields. Minerals containing much of sodium oxide and potassium oxide are colorless and hence only slightly sensitive to visible light or near-infrared radiation. Since the yield of secondary ions correlates closely with the ionization energy (Storms et al., 1977), remote SIMS analysis of the lunar surface would be especially effective in detecting alkali species with lower ionization energies. The variation in alkali abundance is a key to indicating the compositional differences of the magma resource. Alkali oxides have usually been used for grouping the lunar impact-melt breccias with the returned samples (e.g., Korotev, 2000). Remote SIMS measurement in orbit certainly complements $\mathrm{X}$-ray fluorescence and gamma-ray spectroscopy for elemental abundance and multi-band spectral imaging for mineralogy (Elphic et al., 1991).

We have shown that remote SIMS analyses is achievable by measuring picked-up lunar ions at an altitude of $100 \mathrm{~km}$ and can be used to do global composition mapping of the lunar surface. In fact, remote SIMS analysis of the lunar surface is planned in a future Japanese lunar orbiter mission named 'SELENE'. Now we know that the energy and motion direction of picked-up lunar ions at an altitude of 100 $\mathrm{km}$ is virtually determined by the surrounding magnetic and electric fields because the gravity of the Moon and radiation pressure have much less effect on ion motion. Therefore, an ion measurement system onboard a lunar orbiter is able to predict the energy and motion direction of picked-up ions by monitoring the surrounding magnetic and electric fields. By appropriately fixing the selectable energy and field-ofview ranges, the analyzer can make a concentrated detection of the ions. Although general ion mass analyzers for space plasma missions cannot distinguish such heavy ions as $\mathrm{Na}^{+}$, $\mathrm{Mg}^{+}$etc., the ion spectrometer onboard SELENE has mass resolution $(\mathrm{M} / \Delta \mathrm{M} \sim 20)$ that is high enough to distinguish such heavy ions with deconvolution tools (Yokota et al., 2005).

Acknowledgments. The authors would like to thank Dr. H. Jin of the Institute of Space Astronautical Science (ISAS) for his discussion on the simulation method. Sincere thanks are due to Professor T. Mukai of ISAS for his valuable comments. This work was supported in part by Research Fellowships of the Japan Society for the Promotion of Science for Young Scientists.

\section{References}

Ageev, V. N., Yu. A. Kuznetsov, B. V. Yakshinskiy, and T. E. Madey, Electron stimulated desorption of alkali metal ions and atoms: Local surface field relaxation, Nucl. Instr. Meth., B101, 69-72, 1995.

Andersen, C. A. and J. R. Hinthorne, Ion microprobe mass analyzer, Science, 175, 853-860, 1972.

Bavassano, B., M. Dobrowolny, F. Mariani, and N. F. Ness, Radial evolution of power spectra of interplanetary Alfvénic turbulence, J. Geophys. Res., 87, 3616-3622, 1982.

Ben, D., J. Bussey, and P. D. Spudis, Compositional studies of the Orientale, Humorum, Nectaris, and Crisium lunar basins, J. Geophys. Res., 105, 4235-4244, 2000.

Cladis, J. B., W. E. Francis, and R. R. Vondrak, Transport toward earth of ions sputtered from the moon's surface by the solar wind, J. Geophys. Res., 99, 53-64, 1994. 
Coleman, P. J., Jr., Turbulence, viscosity, and dissipation in the solar-wind plasma, Astrophys. J., 153, 371-388, 1968.

Cremonese, G. and S. Verani, High resolution observations of the sodium emission from the Moon, Adv. Space Res., 19(1), 1561-1569, 1997.

Denskat, K. U., H. J. Beinroth, and F. M. Neubauer, Interplanetary magnetic field power spectra with frequencies from $2.4 \times 10$ to the -5 th $\mathrm{HZ}$ to $470 \mathrm{HZ}$ from HELIOS-observations during solar minimum conditions, J. Geophys., 54, 60-67, 1983.

Elphic, R. C., H. O. Funsten, III, B. L. Barraclough, D. J. McComas, M. T. Paffett, D. T. Vaniman, and G. Heiken, Lunar surface composition and solar wind-induced secondary ion mass spectrometry, Geophys. Res. Lett., 18, 2165-2168, 1991.

Feldman, P. D. and D. Morrison, The Apollo 17 ultraviolet spectrometer: Lunar atmosphere measurements revisited, Geophys. Res. Lett., 18(11), 2105-2109, 1991.

Flynn, B. and S. A. Stern, A spectroscopic survey of metallic species abundances in the lunar atmosphere, Icarus, 124, 530-536, 1996.

Freeman, J. W. and M. Ibrahim, Lunar electric fields, surface potential and associated plasma sheaths, Moon, 14, 103-114, 1975.

Funsten, R. C., R. C. Elphic, B. L. Barraclough, D. J. McComas, K. R. Moore, and J. E. Nordholt, Lunar and asteroid composition assessment using remote secondary ion mass spectrometry, Proc. Int. Symp. on Spectral Sensing Res., Maui, HI., 1332-1343, 1992.

Gaddis, L. R., B. R. Hawke, M. S. Robinson, and C. Coombs, Compositional analyses of small lunar pyroclastic deposits using Clementine multispectral data, J. Geophys. Res., 105, 4245-4262, 2000.

Halekas, J. S., D. L. Mitchell, R. P. Lin, S. Frey, L. L. Hood, M. H. Acuña, and A. B. Binder, Mapping of crustal magnetic anomalies on the lunar near side by the Lunar Prospector electron reflectometer, J. Geophys. Res., 106(E11), 27,841-27,852, 2001.

Hilchenbach, M., D. Hovestadt, B. Klecker, and E. Mobius, Observation of energetic lunar pick-up ions near Earth, Adv. Space Res., 13(10), 321324, 1993.

Hodges, R. R., Jr., Formation of the lunar atmosphere, Moon, 14, 139-157, 1975.

Hodges, R. R., Jr., J. H. Hoffman, T. T. J. Yeh, and G. K. Chang, Orbital search for lunar volcanism, J. Geophys. Res., 77(22), 4079-4085, 1972.

Killen, R. M. and W.-H. Ip, The surface-bounded atmospheres of Mercury and the Moon, Rev. Geophys., 37, 361-406, 1999.

Korotev, R. L., The great lunar hot spot and the composition and origin of the Apollo mafic ("LKFM") impact-melt breccias, J. Geophys. Res., 105(E2), 4317-4345, 2000.

Lin, R. P., D. L. Mitchell, D. W. Curtis, K. A. Anderson, C. W. Carlson, J. McFadden, M. H. Acuña, L. L. Hood, and A. Binder, Lunar surface magnetic fields and their interaction with the solar wind: Results from Lunar Prospector, Science, 281, 1480-1484, 1998.

Madey, T. E., B. V. Yakshinskiy, V. N. Ageev, and R. E. Johnson, Desorption of alkali atoms and ions from oxide surfaces: Relative to the origins of $\mathrm{Na}$ and $\mathrm{K}$ in atmospheres of Mercury and the Moon, J. Geophys. Res., 103(E3), 5873-5877, 1998.

Mall, U., E. Kirsch, K. Cierpka, B. Wilken, A. Söding, F. Neubauer, G. Gloeckler, and A. Galvin, Direct observation of lunar pick-up ions near the Moon, Geophys. Res. Lett., 25(20), 3799-3802, 1998.
Managadze, G. G. and R. Z. Sagdeev, Chemical composition of small bodies of the solar system determined from the effects of solar-wind interaction with their surfaces, Icarus, 73, 294-302, 1988.

Mendillo, M., J. Baumgardner and B. Flynn, Imaging observations of the extended sodium atmosphere of the moon, Geophys. Res. Lett., 18, 2097-2100, 1991.

Potter, A. E. and T. H. Morgan, Discovery of the sodium and potassium vapor in the atmosphere of the Moon, Science, 241, 675-679, 1988.

Potter, A. E. and T. H. Morgan, Observation of the lunar sodium exosphere, Geophys. Res. Lett., 18, 2089-2092, 1991.

Potter, A. E. and T. H. Morgan, Coronagraphic observations of the lunar sodium exosphere near the lunar surface, J. Geophys. Res., 103, 85818586, 1998.

Potter, A. E., R. M. Killen, and T. H. Morgan, Variation of lunar sodium during passage of the Moon through the Earth's magnetotail, J. Geophys. Res., 105(E6), 15,073-15,084, 2000.

Price, C. P. and C. S. Wu, The influence of strong hydromangetic turbulence on newborn cometary ions, Geophys. Res. Lett., 14, 856-859, 1987.

Smith, S. M., J. K. Wilson, J. Baumgardner, and M. Mendillo, Discovery of the distant lunar sodium tail and its enhancement following the Leonid meteor shower of 1998, Geophys. Res. Lett., 26, 1649-1652, 1999.

Smyth, W. H. and M. L. Marconi, Theoretical overview and modeling of the sodium and potassium atmospheres of the Moon, Astrophys. J., 443, 371-392, 1995.

Sprague, A. L., R. W. H. Kozlowski, D. M. Hunten, W. K. Wells, and F. A. Grosse, The sodium and potassium atmosphere of the Moon and its interaction with the surface, Icarus, 96, 27-42, 1992.

Stern, S. A., J. W. Parker, T. H. Morgan, B. C. Flynn, D. M. Hunten, A. L. Sprague, M. Mendillo, and M. C. Festou, An HST search for magnesium in the lunar atmosphere, Icarus, 127, 523-526, 1997.

Storms, H. A., K. F. Brown, and J. D. Stein, Evaluation of a cesium positive ion source for secondary ion mass spectrometry, Anal. Chem., 49, 20232030, 1977.

Terasawa, T., Particle scattering and acceleration in a turbulent plasma around comets, in Plasma Waves and Instabilities at Comets and Magnetospheres, Geophys. Monogr. Ser., vol. 53, edited by B. T. Tsurutani and H. Oya, pp. 41-49, AGU, Washington, D. C., 1989.

Tyler, A. L., W. H. Kozlowski, and D. M. Hunten, Observations of sodium in the tenuous lunar atmosphere, Geophys. Res. Lett., 15, 1141-1144, 1988.

Walbrige, E., Lunar Photoelectron Layer, J. Geophys. Res., 77, 3668-3687, 1973.

Yakshinskiy, B. V. and T. E. Madey, Photon-stimulated desorption as a substantial source of sodium in the lunar atmosphere, Nature, 400, 642644, 1999.

Yokota, S., Y. Saito, K. Asamura, and T. Mukai, Development of an ion energy mass spectrometer for application on board three-axis stabilized spacecraft, Rev. Sci. Instrum., 76, 014501-1-8, 2005.

S. Yokota (e-mail: s.yokota@nict.go.jp) and Y. Saito 